\title{
ULTRA SONIC TESTING OF WELDJOINTS PREPARED AT DIFFERENT VOLTAGE AND CURRENT
}

\author{
Vivek Varia ${ }^{1}$, Yash Ganatra ${ }^{2}$ \\ ${ }^{1,2}$ School of Mechanical and Building Sciences, Vellore Institute of Technology \\ varia.vivekvimal@gmail.com,gyash1992@gmail.com
}

\begin{abstract}
There are several millions of weld joints made at every corner of the world. There are chances that safety may be ceased to exist because of some defects in the weld joints. The objective of this investigation was to verify the influence of current and voltage on porosity found inside weld joints and other visual defects. Non - Destructive testing was carried out for standard butt joint from Manual metal arc welding (stick welding) and Gas metal arc welding. Porosity was measured in terms of depth from the surface of weld joint. The results will be presented in this paper with interesting finding with test sample by different welding technique.
\end{abstract}

Keywords: Non-Destructive Testing, Stick Welding, Gas Metal Arc Welding, Butt Joint.

\section{INTRODUCTION}

There are thousands of weld joints made using different welding techniques. Although these welding techniques are well proven there might be possibility of defect which may create a problem. For this reason Ultra-Sonic testing of weld joints is carried out. ${ }^{[1]}$ Welding joints are made setting different parameters like current, voltage and welding speed. This paper primarily focuses on welding current and voltage.

\section{LITERATURE STUDY}

Ultrasonic testing (UT) is a non-destructive inspection method that uses high frequency sound waves (ultrasound) that are above the range of human hearing, to measure geometric and physical properties in materials.[2] Ultrasound travels in different materials at different speeds (velocity). However, the speed of sound propagation in a given material is a constant.[10] There are several ways that sound travels through a material. One type of sound wave, called longitudinal or compression travels about 330 meters per second in air and about 6400 meters.[3]

The elements that are common in every NDT Test are:[9]

a. Some source of probing energy or probing medium

b. Discontinuity that causes a change In the probing medium.

c. Some source of detecting the change

d. Some means of indicating the change

e. Some means of recording the change so that interpretations can be made.

The Ultrasonic testing is based on the fact that solid materials are good conductors of sound waves, whereby they are reflected not only from the surface but also from the internal flaws.[5] The interaction of sound waves with material is stronger when wavelength is smaller. The piezoelectric element excited by an extremely short electrical discharge transmits an ultrasonic pulse. The same element generates an electric signal when it receives an ultrasonic signal. The probe is coupled to the surface with a coupling paste so that the sound waves from the probe are directed into the test object. The part of the sound pulse transmitted though the couplet, generates a small electrical signal which causes a vertical deflection of the beam spot. This is known as the back wall echo. If a discontinuity is present, another pulse is detected between the initial echo and the back wall echo.[6]

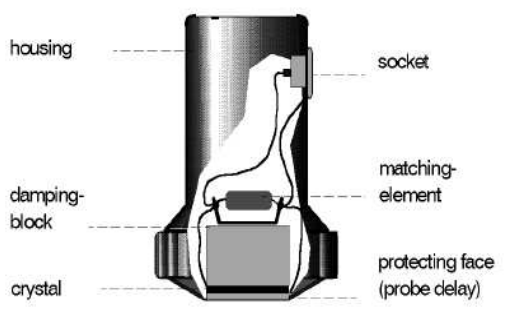

Fig. 1a Straight-beam probe (section)

Fig 1 Ultrasonic testing probe [4] 


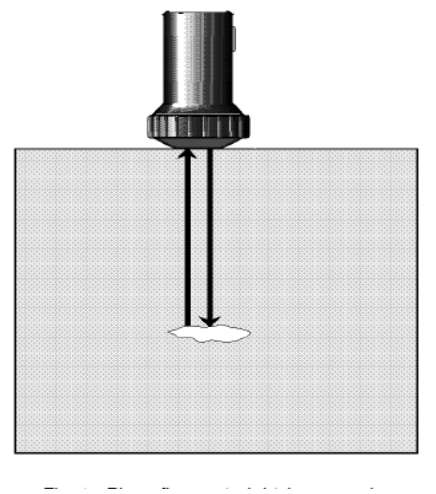

Fig 2 Straight beam probe [4]

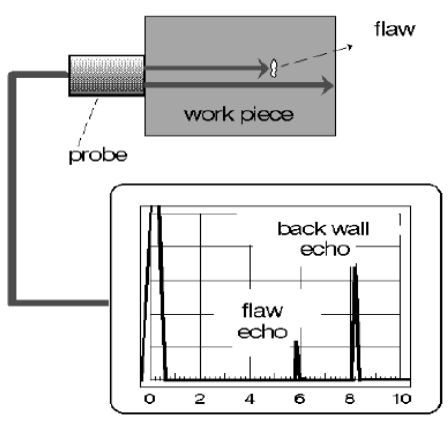

Fig 3 Flaw echo [4]

\section{SETUP}

Equipment parameters:

- Model Name: Einstein II- TFT

- Manufacturer: MODSONIC

- Couplant:

- Zero : 2967

- Range: $50 \mathrm{~mm}$

- Velocity : $5920 \mathrm{~m} / \mathrm{s}$

- Delay: $0 \mathrm{~mm}$

- Gain: $27 \mathrm{~dB}$

The study was carried on standard specimen. The material properties are as follows:

Material: Mild Steel

Thickness: $6 \mathrm{~mm}$

Weld Length: $100 \mathrm{~mm}$

Edge Preparation: Single V Groove

Weld Joint: Butt Joint

The welding joints where prepared using above specimen using Manual metal arc welding and Gas metal arc welding. These joints where prepared using different values of current and voltage.
Table 1: Current settings in stick welding

\begin{tabular}{|l|l|l|}
\hline Voltage (V) & Current (A) & Description \\
\hline 400 & 125 & High Current \\
\hline 440 & 75 & Optimal \\
\hline 425 & 175 & High Voltage \\
\hline
\end{tabular}

Table 2: Voltage settings in mig welding

\begin{tabular}{|l|l|l|}
\hline Voltage(V) & Current $(\mathrm{A})$ & Description \\
\hline 31 & 50 & Normal \\
\hline 25 & 30 & Low Voltage \\
\hline 69 & 20 & High Voltage \\
\hline
\end{tabular}

\section{RESULT}

The defect size as well as the number is least in the middle portion[11]. In Manual Metal Arc Welding, the flaws are obtained nearer to the surface as compared to gas metal arc welding which indicates that penetration is more in MIG Welding. Maximum defects are observed at the start. This can be due to the arc blowing effect wherein the arc is not stable. High current results in more spatter. High Voltage results in sticking of the arc to the base metal. Maximum defects are generally observed at the end. Also when current s high sputtering on the surface also increases.[7]

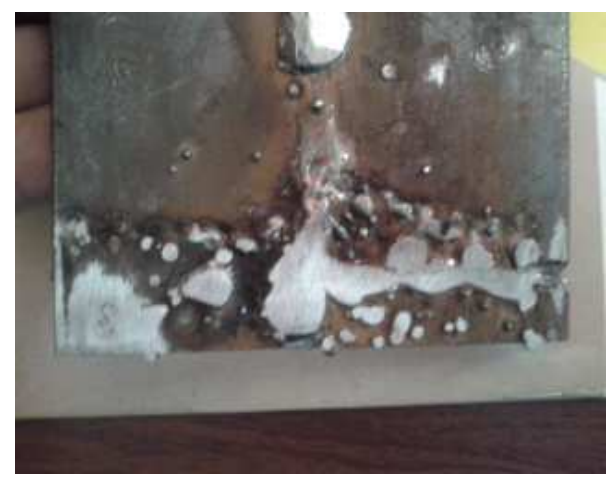

Fig 4 Mig high current 


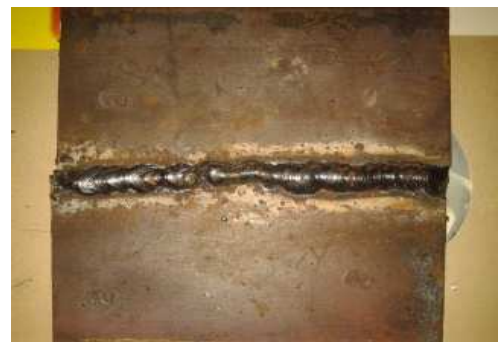

Fig 5 Stick normal current

\subsection{Manual Metal Arc Welding:}

\subsubsection{Normal Current:}

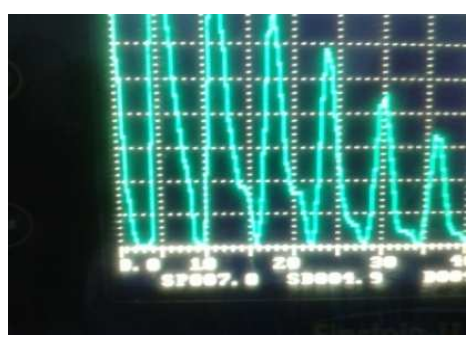

Fig 6 Ultrasonic pattern at start

The defect is present at a depth of $2 \mathrm{~mm}$. The amplitude of the echo is the highest at the start. This implies that the size of the defect is maximum

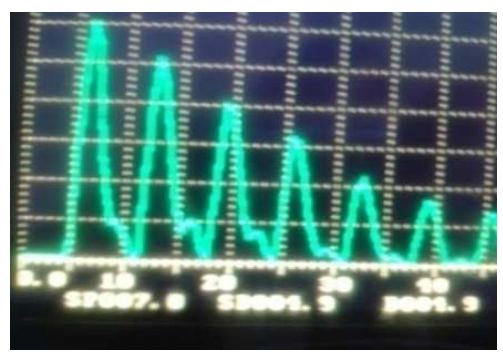

Fig 7 Ultrasonic pattern at middle

At the middle, the defect is detected at a depth of $5 \mathrm{~mm}$. The amplitude of the echo is lesser.

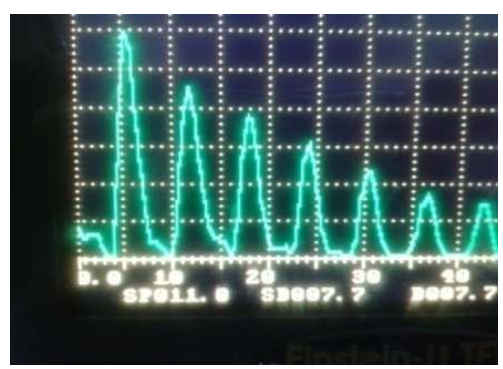

Fig 8 Ultrasonic pattern at end
The discontinuity is detected at $5 \mathrm{~mm}$. The size of the discontinuity is smaller than that obtained in the beginning.

\subsubsection{High Current:}

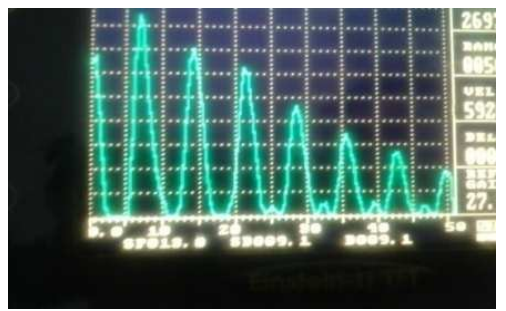

Fig 9 Ultrasonic pattern at start

The defect is detected at a depth of $2 \mathrm{~mm}$.

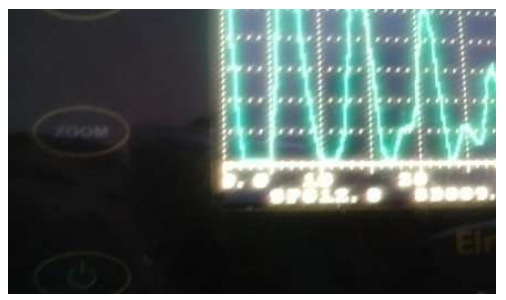

Fig 10 Ultrasonic pattern at middle

The defect is detected at a depth of $3 \mathrm{~mm}$. The size of the defect is smaller than that obtained at the start.

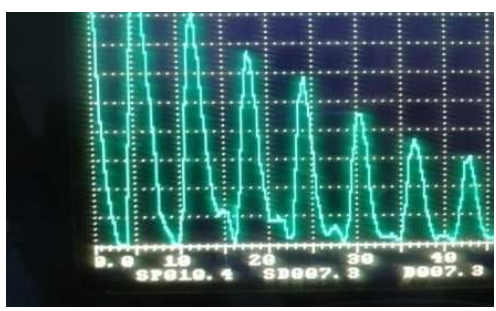

Fig 11 Ultrasonic pattern at end

The flaw is at a depth of $5 \mathrm{~mm}$ but the echo amplitude suggests that the size of the discontinuity is significantly higher.

\subsubsection{High Voltage:}

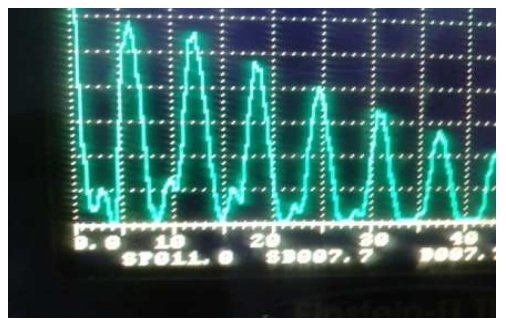

Fig 12 Ultrasonic pattern at start 
The flaw is at a depth of $5 \mathrm{~mm}$ but the echo amplitude suggests that the size of the discontinuity is lower than obtained in earlier two cases.(Normal and High Current)

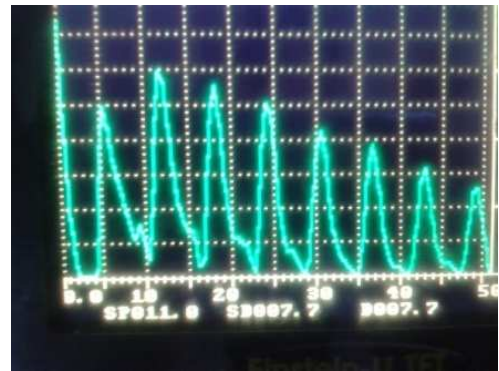

Fig 13 Ultrasonic pattern at middle

The flaw is at a depth of $4 \mathrm{~mm}$ but the echo amplitude suggests that the size of the discontinuity remains almost same.

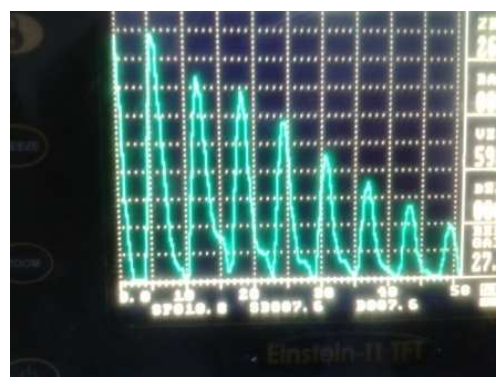

Fig 14 Ultrasonic pattern at end

The flaw is at a depth of $4 \mathrm{~mm}$ but the echo amplitude suggests that the size of the discontinuity has increased significantly. This can be due to the sticking of the arc.

\subsection{Gas Metal Arc Welding (MIG):}

\subsubsection{Normal Current}

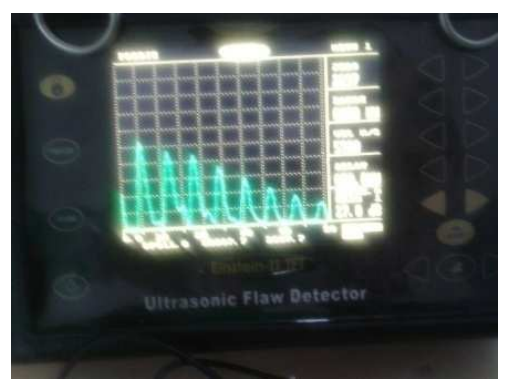

Fig 15 Ultrasonic pattern at start

The discontinuity is obtained at $5 \mathrm{~mm}$. The size of discontinuity is less than those obtained in Manual Metal Arc Welding. Gas Metal Arc Welding provides better control and precision.

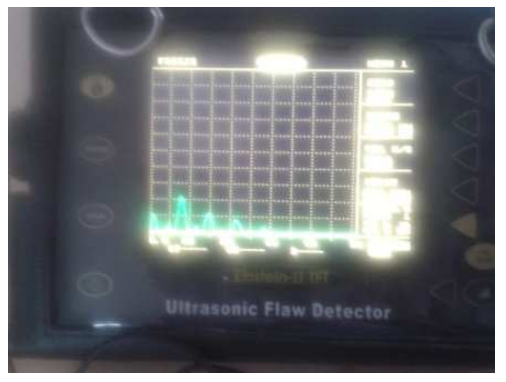

Fig 16 Ultrasonic pattern at middle

The flaw is detected at a depth of $5 \mathrm{~mm}$. The amplitude of the echo is less which implies that the size of discontinuity is less. Also, this indicates a complete penetration.

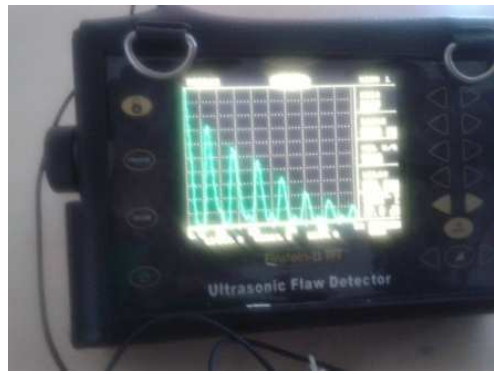

Fig17 Ultrasonic pattern at end

The flaw is detected at a depth of $3 \mathrm{~mm}$. The amplitude of the echo is more which implies that the size of discontinuity is more.

\subsubsection{High Current:}

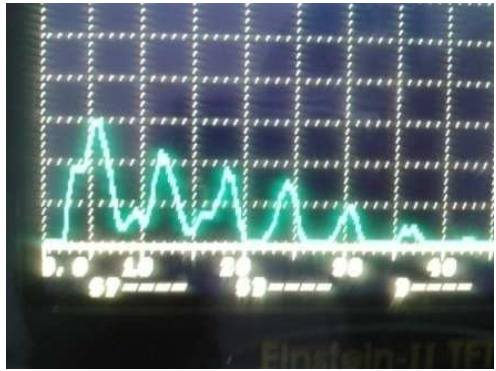

Fig 18 Ultrasonic pattern at start

The flaw is detected at a depth of $3 \mathrm{~mm}$. The amplitude of the echo is less which implies that the size of discontinuity is less. 


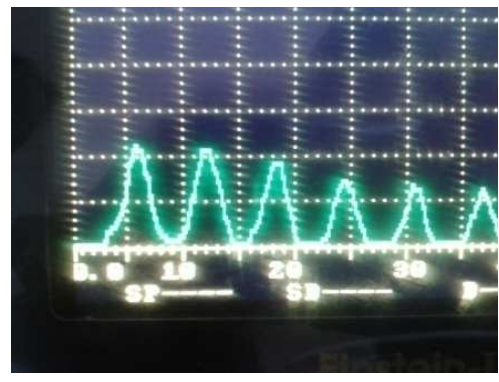

Fig 19 Ultrasonic pattern at middle

The flaw is detected at a depth of $3 \mathrm{~mm}$.

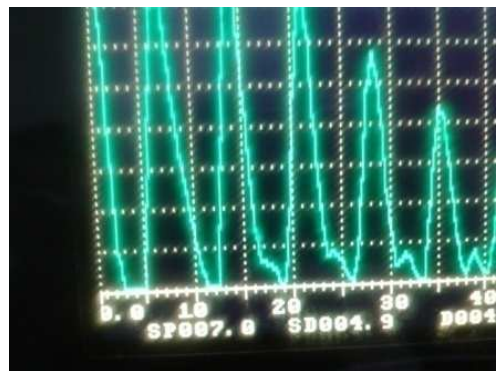

Fig 20 Ultrasonic pattern at end

The flaw is at a depth of $4 \mathrm{~mm}$ but the echo amplitude suggests that the size of the discontinuity has increased significantly.

\section{CONCLUSIONS}

Ultrasonic Testing of $6 \mathrm{~mm}$ thick mild steel plates welded using Stick and Gas Metal Arc Welding was done. Different defects are observed.[8] Maximum defects are observed when high current and high voltage is used. With high current the sputtering is also increased. Different materials have different current-voltage characteristics. Amplitude Reject Level (ARL) and Disregard Level (DRL) indicate the set levels for acceptance and rejection of amplitude. Different techniques for categorization of defects involve use of Neural Networks and Data Acquisition and filtering techniques. Genetic Algorithms are being developed. [7]

\section{REFERENCES}

[1] Non Destructive Material Testing with UltrasonicsKrautkramer.

[2] Non Destructive Testing - Det Norske Veritas (DNV), March 2012

[3] A Guide for Ultrasonic Testing and Evaluation of flaws - Ship Structure Committee, USA, 1970

[4] Bredif P., Plu J., Pouligny P. and Poldevin C. Phasedarray method for the us-inspection of French rail repairs. CP975. Review of Quantitative Nondestructive Evaluation. Ed by D. O.Thompson and
E.Chimenti. 2008. Vol.27. American Institute of Physics 978-0-7354-0494-6/08

[5] M. Berke, U. Hoppenkamps: "Testing materials ultrasonically" Krautkrämer Training System, Level 1 3rd edition (1990)

[6] The ultrasonic wave interaction with porosity defects in welded rail head E. Jasiūnienè, E. Žukauskas ISSN 1392-2114

[7] WELDING DEFECTS PATTERN RECOGNITION IN TOFD SIGNALS USING LINEAR CLASSIFIER IMPLEMENTED BY NEURAL NETWORKS Elineudo P. de Moura

[8] Silk, M.G., LIDINGTON, B.H., "Defect sizing using an Ultrasonic Time Delay Approach", British Journal of NDT, pp. 33-36, March, 1975

[9] Defects Clustering using Kohonen Networks during Ultrasonic Inspection Thouraya Merazi Meksen, Bachir Boudraa, Malika Boudraa

[10] Non Destructive Testing Handbook. American Society for Nondestructive Testing, USA, 1986.

[11] T. Stepinski, F. Lingvall, Automated Defect Characterization in Ultrasonic NDT, 15th WCNDT, Roma, 2000 